\title{
Demirtaş Erciyes-Mid urethral fibrin fixation technique (DE-MUFFT) for female stress urinary incontinence: A case series
}

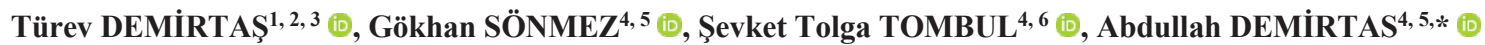

${ }^{1}$ Department of Medical History and Ethics, Erciyes University, Kayseri, Turkey

${ }^{2}$ Department of Stem Cell Sciences, Genom and Stem Cell Center, Erciyes University, Kayseri, Turkey

${ }^{3}$ Department of Emergency Medical Program, Halil Bayraktar Vocational Health Collage, Erciyes University, Kayseri, Turkey

${ }^{4}$ Department of Urology, Erciyes University, Kayseri, Turkey

${ }^{5}$ Department of Molecular Biology and Genetics, Genom and Stem Cell Center, Erciyes University, Kayseri, Turkey

${ }^{6}$ Department of Pharmaceutical Research, Development and Application, Erciyes University, Kayseri, Turkey

\begin{tabular}{ccccc}
\hline Received: 03.04.2021 $\quad$ Accepted/Published Online: 23.04 .2021 & • & Final Version: 30.08 .2021 \\
\hline
\end{tabular}

\begin{abstract}
Factors including suburethral blood flow impairment, collagen deficiency, and the lack of tissue healing factors are known to play a role in stress urinary incontinence (SUI). Autologous fibrin (AF) appears to be a viable material for the treatment of SUI. The aim of this study was to present the initial clinical outcomes of a novel technique named "Demirtaş Erciyes-Mid Urethral Fibrin Fixation Technique (DE-MUFFT)" that involved the placement of AF material in the suburethral space instead of sling material (mesh). In this study, the clinical outcomes of five women with pure SUI who underwent the placement of AF material in the suburethral space were examined retrospectively. The complaint of urine leakage during physical exertion and patients' quality of life were assessed using Incontinence Quality of Life Scale (I-QoL), International Consultation on Incontinence Questionnaire-Urinary Incontinence Short Form (ICIQ-UI SF), Incontinence Impact Questionnaire (IIQ-7), and 24-h pad test. Patient outcomes were evaluated preoperatively, at sixth week and third month postoperatively. Significant improvement was obtained in the quality-of-life tests and 24-h pad test. In all patients, the complaint of urine leakage disappeared almost completely. No adverse event or postoperative complication occurred in any of the patients. These results indicated that DE-MUFFT can be a promising procedure in the treatment of SUI due to its biocompatibility, minimally invasive nature, re-applicability, and cost-effectivity.
\end{abstract}

Keywords: autologous fibrin, platelet rich fibrin, stress urinary incontinence, sling

\section{Introduction}

Stress urinary incontinence (SUI) is a commonly seen health problem, affecting almost half of adult women with urinary incontinence (1). Despite their complications (erosion, infection, obstruction) and high costs, minimally invasive sling procedures such as transobturator tape (TOT) and transvaginal tape (TVT) are the most common surgical techniques used in the treatment of SUI (2). The primary aim in these techniques is to support the urethra and bladder neck with a synthetic mesh (3). In addition to the suspension mechanism formed by the sling, some researchers advocate that the inflammation, increased blood supply, and tissue reactions in the region where the sling is placed also support the urethra and thus provide clinical benefit (4-8).

Autologous Fibrin (AF) is a fibrin matrix frequently used in the treatment of clinical and urological diseases, consisting of cytokines, growth factors, and stem cell-like cells that are essential for immunomodulation and tissue healing $(9,10)$. To date, some other techniques such as stem cell injection, platelet rich plasma (PRP), and laser ablation have also been used for forming local inflammation and tissue response (4, 5,
11). However, to our knowledge, the use of AF in the treatment of SUI has not been reported in the literature.

The aim of this study is to describe a novel technique, which named as Demirtaş Erciyes-Mid Urethral Fibrin Fixation Technique (DE-MUFFT)", involved the placement of AF material in the suburethral space as the tissue support material without using a mesh-like foreign body and to present the clinical outcomes of the five patients with pure SUI that underwent this technique in our clinic.

\section{Materials and Methods}

\subsection{Urethral preparation}

In the lithotomy position, a 14-16 F Foley catheter is placed in the urethra. The balloon is inflated with $15 \mathrm{ml}$ fluid and is placed against the bladder neck. The location of the bladder neck is determined and marked by moving the catheter in different directions. A 2-cm midline vertical incision is performed in the anterior vaginal wall, beginning $5 \mathrm{~mm}$ proximal to the urethral meatus, and extending to the bladder neck (Fig. 1A). Subsequently, sharp, and blunt dissection is used at the level of inferior pubic ramus, superior to the 
endopelvic fascia, to release the urethra (excluding the dorsal aspect) by preserving the periurethral tissue (Fig. 1B).

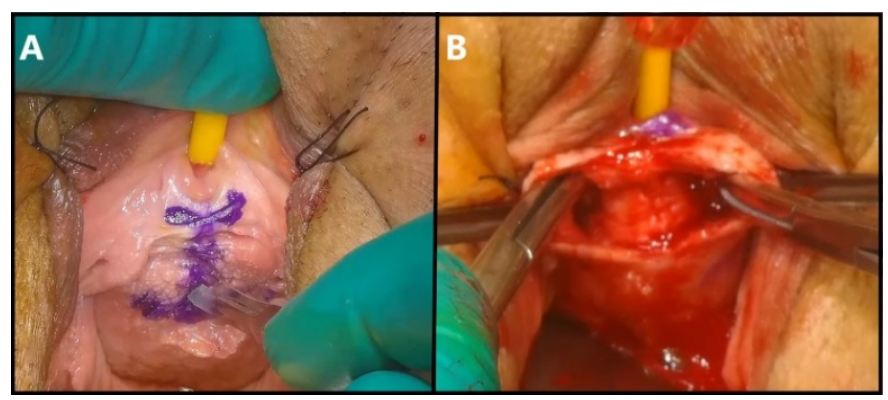

Fig. 1. Urethral preparation. A. 2-cm midline vertical incision, B. sharp and blunt dissection from the sides of the urethra

\subsection{Acquisition of $\mathbf{A F}$}

A total of $20 \mathrm{ml}$ of peripheral venous blood sample is placed into 4 biochemical tubes with separator gels, hence equally divided into 4 portions (Fig. 2A). The tubes are centrifuged at 2,700 rpm for $12 \mathrm{~min}$ (Fig. 2B) (12). The AF material that often forms in the middle of the tube is separated from the clot that forms at the bottom of the tube and then placed on a gauze pad (Fig. 3A). Afterwards, all the obtained AF materials are placed between two saline-soaked gauze pads either in combination or separately and transformed to membrane by minimal finger pressure (Fig. 3B).

\subsection{Insertion of $\mathbf{A F}$ and finalization of the procedure}

The AF membranes are inserted in the space previously formed below the urethra and then fixed to both ends of the external urethral meatus using 4-0 absorbable sutures (Fig. $4 \mathrm{~A}$ and $4 \mathrm{~B}$ ). At this point, care should be taken to avoid the passage of the suture into the urethral lumen. The procedure is completed by closing the subcutaneous and skin layers with absorbable sutures. One of the four AF membranes is placed on the right, one on the left, and the other two in the middle suburethral space.

\subsection{Data Collection}

The clinical outcomes of five women with pure SUI who underwent DE-MUFFT were examined. The complaint of urine leakage during physical exertion and patients' quality of life were assessed using Incontinence Quality of Life Scale (IQoL), International Consultation on Incontinence Questionnaire-Urinary Incontinence Short Form (ICIQ-UI $\mathrm{SF}$ ), Incontinence Impact Questionnaire (IIQ-7), and 24-h pad test. Patient outcomes were evaluated preoperatively, at sixth week and third month postoperatively.

\section{Results}

The mean age of the patients included in the study was $53.2 \pm$ 3.7 years. The mean duration of SUI complaints was $26.0 \pm$ 4.5 months, and the mean postoperative follow-up time was $17.0 \pm 3.9$ months. All patients had previously applied pelvic floor exercises and used medical therapy (oral duloxetine) but did not benefit from these treatments. Urogenital examinations showed no clinically significant cystocele, rectocele, or descensus. The uroflowmetric parameters (maximum flow rate and post-voiding residual urine) of the patients were normal in pereoperatively and postoperatively.

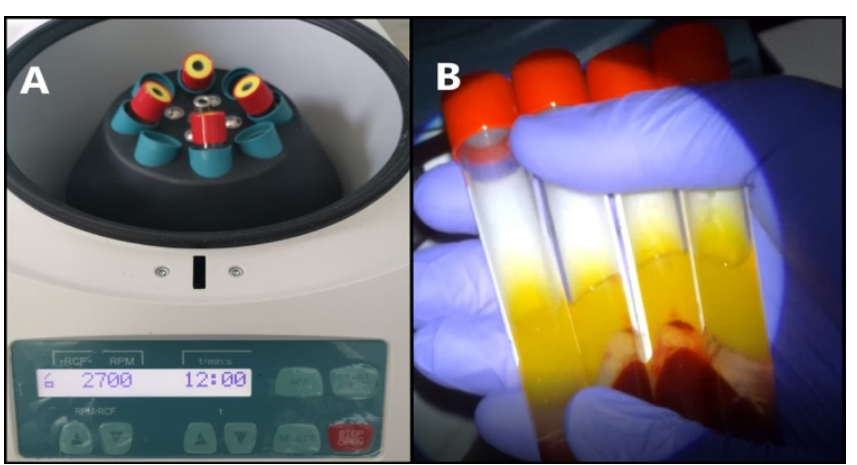

Fig. 2. Acquisition of AF (Part-1). A. The tubes are centrifuged at 2,700 rpm for $12 \mathrm{~min}, \mathbf{B}$. The AF material that forms in the middle of the tube

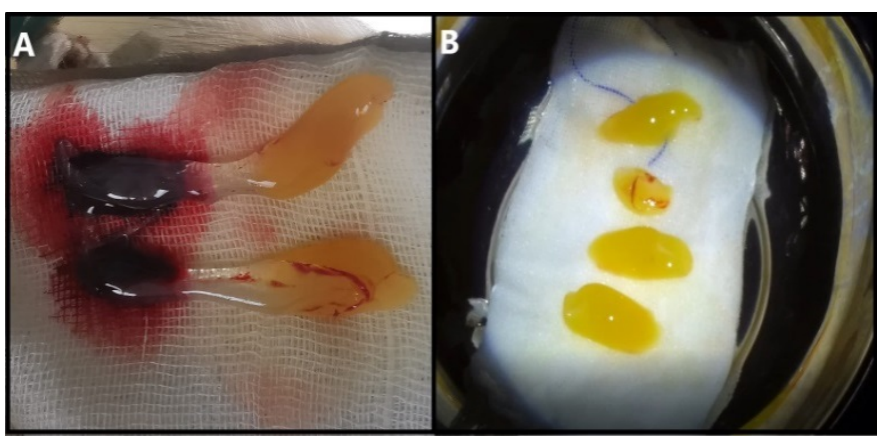

Fig. 3. Acquisition of AF (Part-2). A. The AF material is separated from the clot that forms at the bottom of the tube and then placed on a gauze pad. B. AF fragments separated from other cells and ready for processing

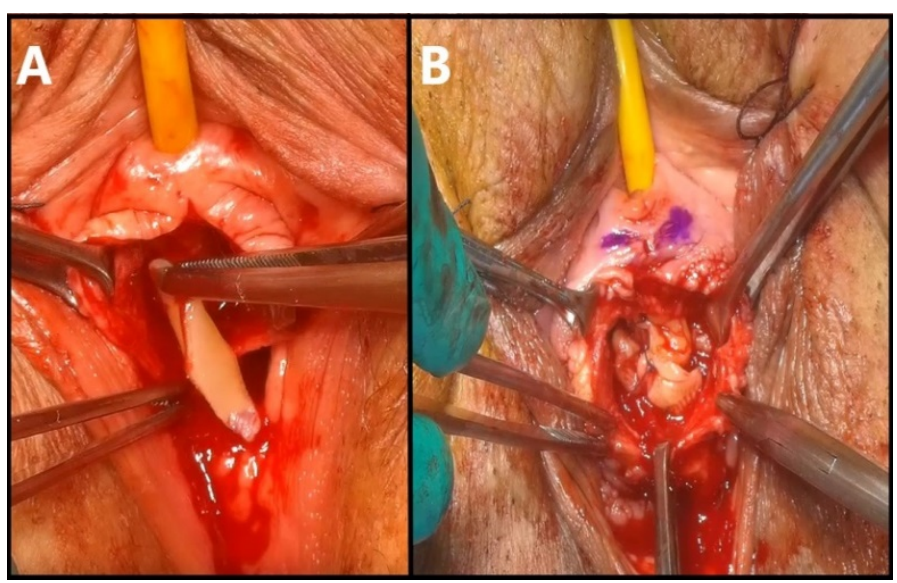

Fig.4. Insertion of AF. A. The AF membranes are inserted in the space previously formed, B. The AF membranes are fixed to both ends of the external urethral meatus using 4-0 absorbable sutures

In all patients, at six-week follow-up, urine leakage during sudden physical exertion and physical exercise reduced remarkably, I-QoL, ICIQ-UI/SF, IIQ-7 scores showed improvement, and the $24-\mathrm{h}$ pad tests were negative.

In one of the five patients who completed their 12-week follow-up, it was observed that SUI symptoms started again at a very mild level. Despite this, the patient's satisfaction rate was $90 \%$. The other patients did not have any SUI symptoms. Preoperatively, postoperative 6th and 12th week SUI tests of the patients are shown in Table 1, and their questionnaires are shown in Table 2. 
Table 1. Demographics and SUI tests of the cases

\begin{tabular}{c|c|c|c|c|c|c|c|c}
\hline Case & Age & Follow-up time (weeks) & ST-0 & ST-6 & ST-12 & PT-0 & PT-6 & PT-12 \\
\hline$\# \mathbf{1}$ & 54 & 21 & + & - & - & 4 pads/day & 0 & 0 \\
\hline$\# \mathbf{2}$ & 59 & 20 & + & - & + & 3 pads/day & 0 & $\mathbf{1}$ \\
\hline $\mathbf{3}$ & 49 & 18 & + & - & - & 4 pads/day & 0 & 0 \\
\hline $\mathbf{4}$ & 51 & 14 & + & - & - & 5 pads/day & 0 & 0 \\
\hline$\# \mathbf{5}$ & 53 & 12 & + & - & - & 5 pads/day & 0 & 0 \\
\hline
\end{tabular}

ST-0: Stress test-preoperative, ST-6: Stress test-sixt week, ST-12: Stress test-third month, PT-0: 24 hours' pad test-preoperative, PT-6: 24 hours' pad test-sixt week, PT-12: 24 hours' pad test-third month

At $24^{\text {th }} \mathrm{h}$ after the AF procedure, the patients were had early or late postoperative complications. discharged following catheter removal. None of the patients

Table 2. Questionnaire results of the cases

\begin{tabular}{c|c|c|c|c|c|c|c|c|c|c|}
\hline Case & I-QoL-0 & I-QoL-6 & I-QoL-12 & ICIG-UI-0 & ICIG-UI-6 & ICIG-UI-12 & IIQ/7-0 & IIQ/7-6 & IIQ/7-12 \\
\hline$\# \mathbf{1}$ & 39 & 90 & 90 & 16 & 6 & 6 & 15 & 7 \\
\hline \#2 & 42 & 85 & 75 & 18 & 4 & 10 & 16 & 5 \\
\hline \#3 & 27 & 85 & 85 & 10 & 5 & 4 & 15 & 3 \\
\hline \#4 & 41 & 90 & 88 & 14 & 4 & 4 & 18 & 4 \\
\hline$\# \mathbf{5}$ & 35 & 88 & 85 & 15 & 4 & 4 & 14 & 3 \\
\hline
\end{tabular}

I-QoL-0: Incontinence Quality of Life-preoperative, I-QoL-6: Incontinence Quality of Life-sixth week, I-QoL-12: Incontinence Quality of Life-third month, ICIG-UI-0: Consultation on Incontinence Questionnaire Short Form-preoperative, ICIG-UI-6: Consultation on Incontinence Questionnaire Short Form-sixth week, ICIG-UI-12: Consultation on Incontinence Questionnaire Short Form-third month, IIQ/7-0: Incontinence Impact Questionnaire-preoperative, IIQ/7-6: Incontinence Impact Questionnaire-6 ${ }^{\text {th }} \mathrm{wk}$, IIQ/7-6: Incontinence Impact Questionnaire-12 ${ }^{\text {th }} \mathrm{wk}$.

\section{Discussion}

In this study, early postoperative outcomes of five women with pure SUI that underwent DE-MUFFT were highly promising. Although the sling and deep tissue fixation achieved in the technique achieved in the present study were not as substantial as those achieved by TVT and TOT, the patients obtained favorable clinical outcomes, which could be associated with the fibrosis that occurred secondary to suburethral incision and with the increased blood supply, collagen changes, and tissue healing resulting from the insertion of AF membrane.

This physiopathology has also been advocated by some other studies in the literature $(8,13,14)$. In a previous study, Chen et al. evaluated the placement of a vaginal suburethral sling in rats and reported that the histologic changes caused by the insertion of the suburethral sling, such as inflammation, localized edema, and differential collagen remodeling, showed anti-incontinence effects despite the cutting of the polypropylene sling and the deactivation of the sling function (8). Klinge et al. reported that the collagen tissue dysfunction in the suburethral space results in SUI and the authors also confirmed the findings reported by Chen et al (13). Dobberfuhl et al. suggested that the obstruction formed by mid-urethral slings was not mandatory for obtaining successful outcomes (14). On the other hand, AF has been shown to be a viable material for tissue healing, collagen synthesis, fibrosis, and increased blood supply (15). Considering these notions, we consider that the favorable effects of suburethral placement of AF membrane on SUI could explain the physiopathology of the outcomes obtained in our study.
Both PRP and AF have been extensively used in urological diseases. Nikolopoulos et al. injected the PRP material in the pubourethral ligament (PUL) in SUI patients and found that the injection restored the ligament and provided successful outcomes (16). Similarly, Shirvan et al. reported that the closure of the fistula tract with $\mathrm{AF}$ membrane in vesicovaginal fistula repair provided favorable outcomes in the treatment of the fistula (17). A case report published in 2018 indicated that the injection of PRP material in the sphincter in a SUI patient led to $50 \%$ reduction in pad usage (11). The present case series, to our knowledge, is the first study reporting on the insertion of AF membrane in the suburethral region in SUI patients.

The technique administered in the present study provided several key advantages. First, the requirement of mesh, which is a foreign body for the patient, was eliminated. In the literature, mesh-related complications and the requirement of mesh revision surgery have been reported in up to $20 \%$ of the patients (18). Second, the technique was minimally invasive as it required a small suburethral incision and did not require deep tissue fixation as in TOT and TVT. In this way, the technique eliminated the risk of injury to the bladder, urethral, and bowel, which has been reported in up to $4 \%$ of the patients undergoing TVT and TOT procedures. Moreover, the risk of severe postoperative pain, which is a major complication of sling procedures, is remarkably low in the sub urethral AF procedure (18). Accordingly, we believe that this minimally invasive technique can be administered under local anesthesia in daily clinical practice in line with the accumulating experience, albeit not yet administered in our clinic. Third, this technique is highly cost-effective. A recent 
report published in the $\mathrm{UK}$ indicated that mesh-related complications (infection, revision) lead to a significant increase in the costs of sling procedures (19). In contrast, the AF membrane is highly cost-effective since it is obtained from autologous blood sample. Additionally, it is also costeffective when compared to other sling procedures as it does not require postoperative cystoscopy. Fourth, this technique is re-applicable as it solely consists of an autologous and biological material, can be inserted with a minimally invasive procedure, and is relatively cost-effective.

Our study was limited since the technique was performed in five patients only, the long-term outcomes of the patients remained unknown, and the immunohistochemical and histological changes were not analyzed.

In conclusions, DE-MUFFT can be a promising procedure in the treatment of stress urinary incontinence due to its biocompatibility, minimally invasive nature, re-applicability, and cost-effectivity. Further large-scale experimental and randomized-controlled studies based on histological analysis are needed to substantiate our findings.

\section{Conflict of interest}

None.

\section{Acknowledgments}

There is no financial support for this study.

\section{References}

1. Irwin DE, Kopp ZS, Agatep B, Milsom I, Abrams P. Worldwide prevalence estimates of lower urinary tract symptoms, overactive bladder, urinary incontinence and bladder outlet obstruction. BJU Int. 2011;108: 1132-1138.

2. Ross S, Robert M, Lier D, Eliasziw M, Jacobs P. Surgical management of stress urinary incontinence in women: safety, effectiveness and cost-utility of trans-obturator tape (TOT) versus tension-free vaginal tape (TVT) five years after a randomized surgical trial. BMC Womens Health. 2011;11: 34

3. Park KK, Kim SD, Huh JS, Kim YJ. A study of clinical predictors associated with intrinsic sphincter deficiency in women with stress urinary incontinence. Int Neurourol J. 2017;21: 139-42.

4. Okui N. Comparison between erbium-doped yttrium aluminum garnet laser therapy and sling procedures in the treatment of stress and mixed urinary incontinence. World J Urol. 2019;37: 885-889.

5. Zambon JP, Williams KJ, Bennington J, Badlani GH. Applicability of regenerative medicine and tissue engineering for the treatment of stress urinary incontinence in female patients. Neurourol Urodyn. 2019;38: 76-83.

6. Greiman A, Kielb S. Revisions of mid urethral slings can be accomplished in the office. J Urol. 2012;188: 190-3.

7. Boukerrou M, Boulanger L, Rubod C, Lambaudie E, Dubois P, Cosson M. Study of the biomechanical properties of synthetic mesh implanted in vivo. Eur J Obstet Gynecol Reprod Biol. 2007;134: 262-267.

8. Chen CC, Hijaz A, Drazba JA, Damaser MS, Daneshgari F. Collagen remodeling and suburethral inflammation might account for preserved anti-incontinence effects of cut polypropylene sling in rat model. Urology. 2009;73: 415-420.

9. Miron RJ, Zhang Y. Autologous liquid platelet rich fibrin: A novel drug delivery system. Acta Biomater. 2018;75: 35-51.

10. Say F, Buyukceran I, Coskun S. Two different regenerative injections in patients diagnosed with secondary hip osteoarthritis: A report of two cases. J Exp Clin Med. 2021;38: 204-207.

11. Matz EL, Pearlman AM, Terlecki RP. Safety and feasibility of platelet rich fibrin matrix injections for treatment of common urologic conditions. Investig Clin Urol. 2018;59: 61-65.

12. Miron RJ, Chai J, Zheng S, Feng M, Sculean A, Zhang Y. A novel method for evaluating and quantifying cell types in platelet rich fibrin and an introduction to horizontal centrifugation. J Biomed Mater Res A. 2019;107: 2257-2271.

13. Klinge U, Binneboesel M, Kuschel S, Schuessler B. Demands and properties of alloplastic implants for the treatment of stress urinary incontinence. Expert Rev Med Devices. 2007;4: 349-359.

14. Dobberfuhl AD, De EJ. Female stress urinary incontinence and the mid-urethral sling: is obstruction necessary to achieve dryness? World J Urol. 2015;33: 1243-1250.

15. Karimi K, Rockwell H. The Benefits of Platelet-Rich Fibrin. Facial Plast Surg Clin North Am. 2019;27: 331-340.

16. Nikolopoulos KI, Pergialiotis V, Perrea D, Doumouchtsis SK. Restoration of the pubourethral ligament with platelet rich plasma for the treatment of stress urinary incontinence. Med Hypotheses. 2016;90: 29-31.

17. Shirvan MK, Alamdari DH, Ghoreifi A. A novel method for iatrogenic vesicovaginal fistula treatment: autologous platelet rich plasma injection and platelet rich fibrin glue interposition. $\mathrm{J}$ Urol. 2013;189: 2125-2129.

18. Carter P, Fou L, Whiter F, Delgado Nunes V, Hasler E, Austin C, et al. Management of mesh complications following surgery for stress urinary incontinence or pelvic organ prolapse: a systematic review. BJOG. 2020;127: 28-35.

19. Brazzelli M, Javanbakht M, Imamura M, Hudson J, Moloney E, Becker F, et al. Surgical treatments for women with stress urinary incontinence: the ESTER systematic review and economic evaluation. Health Technol Assess. 2019;24: 1-306. 